\title{
Quantum gravity corrections to neutrino propagation
}

\author{
Luis Urrutia* \\ Departamento de Física de Altas Energías, Instituto de Ciencias Nucleares \\ Universidad Nacional Autónoma de México, A.P. 70-543, 04510 México D.F. \\ E-mail: urrutia@nuclecu.unam.mx
}

ABstract: Massive spin-1/2 fields are studied in the framework of loop quantum gravity by considering a state approximating, at a length scale $\mathcal{L}$ much greater than Planck length, a spin- $1 / 2$ field in flat spacetime. The discrete structure of spacetime at $\ell_{P}$ yields modifications to the field propagation at scale $\mathcal{L}$. Neutrino bursts $\left(\bar{p} \approx 10^{5} \mathrm{GeV}\right)$, accompaniying gamma ray bursts, that have travelled distances $L \approx 10^{10}$ light years (ly) are considered. The dominant correction is helicity independent and leads to a time delay of order $\left(\bar{p} \ell_{P}\right) L / c \approx 10^{4} s$. To next order in $\bar{p} \ell_{P}$, the correction has the form of the Gambini and Pullin effect for photons.

A $S$ HAS BEEN recently suggested in the literature [1], [2], [3], quantum gravity effects might be at the edge of observability. One of the proposed ideas is to look for modified dispersion relations of photons with energy $E$ and momentum $\vec{p}$, of the form

$$
c^{2} \vec{p}^{2}=E^{2}\left(1+\xi \frac{E}{E_{Q G}}+O\left(\frac{E}{E_{Q G}}\right)^{2}\right),
$$

where $\xi$ is a parameter of order one and $E_{Q G}$ is a grand unified energy scale of order $\leq 10^{19}$ $\mathrm{GeV}$. The above expression leads to the following modification of the velocity in light propagation

$$
v=\frac{\partial E}{\partial p}=c\left(1-\xi \frac{E}{E_{Q G}}+O\left(\frac{E}{E_{Q G}}\right)^{2}\right),
$$

which implies an uncorrected retardation time $\Delta t$, with respect to pulse propagation with velocity $c$, given by

$$
\Delta t \approx \xi \frac{E}{E_{Q G}} \frac{L}{c}
$$

For cosmological distances $L \approx 10^{10}$ ly and $E_{Q G}$ $\approx 10^{19} \mathrm{GeV}$, the corresponding numbers are $\Delta t \approx$ $10^{-3} \mathrm{~s}$, for $E \approx 20 \mathrm{MeV}$ and $\Delta t \approx 10^{-5} \mathrm{~s}$, for $E \approx 0.20 \mathrm{MeV}$. To detect such effects, an experimental time resolution $\delta t$ less than $\Delta t$ is required

*J. Alfaro and H. Morales-Técotl
Recent observational facts pointing towards the possibility of measuring such effect are: (i) some Gamma Ray Bursts (GRB) originate at cosmological distances, $\left(\sim 10^{10} \mathrm{ly}\right)$ [4]. (ii) sensitivities $\delta t$ up to submillisecond scale have been achieved in recent GRB observations [5]. Sensitivity will be improved with HEGRA and Whipple air Cerenkov telescopes and by AMS and GLAST spatial experiments.

The above points open up the possibility to probe fundamental laws of physics at energy scales near to Planck energy $E_{P}=1.2 \times 10^{19} \mathrm{GeV}$. Indeed, quantum gravity effects could be at the edge of observability $[1,2,3]$.

Modifications to Maxwell's equations in vacuum, induced by quantum gravity effects, have been calculated by Gambini and Pullin [6], using the canonical loop approach to quantum gravity and by Ellis et al.[7], using string theory methods. The former approach leads to the following dispersion relation

$$
\omega_{ \pm}(k)=|k|\left(1 \mp 2 \xi \ell_{P}|k|\right),
$$

where \pm refers to the helicities of the photon and $\ell_{P} \approx 10^{-33} \mathrm{~cm}$ is the Planck length. This parity violating modification predicts helicity dependent birrefingence effects.

On the other hand, in Ref. [7], a string theory aproach suggests that a D-brane recoil in 
the quantum-gravitational foam induces a distortion in the surrounding space, which modifies the propagation properties. The corresponding dispersion relation is

$$
\omega(k)=|k|\left(1-\xi \frac{k}{M_{D}}\right),
$$

with $\xi>0$. This parity conserving modification to Maxwell's equations predicts a first order helicity independent effect in the dispersion relation, which is linear in the photon energy. At this level of the approximation, no birrefringence effects are present. In this approach, the red-shifted difference in the time arrival of two fotons with present day energies $E_{1}$ and $E_{2}$ has been calculated. For the BATSE data, when the redshifts $z$ of the GRB are known, a small subset of coincident photon pulses corresponding to channel 1 (20-50 keV) and chanel 3 (100-300 keV) are fitted and $\Delta t$ is calculated [7]. No significant effect in the data available is found. On the other hand, none of the pulses studied exhibited a microburts structure on short time scales $\left(\leq 10^{-2}\right.$ $\mathrm{s})$. Were this the case, the sensitivity of the analysis would be greatly improved.

Neutrinos could also provide an excellent arena to probe quantum gravity induced propagation effects. In fact, the most widely accepted model of GRB, so called fireball model, predicts the generation of $10^{14}-10^{19} \mathrm{eV}$ Neutrino Bursts (NB) $[8,9]$. Also, the planned Neutrino Burster Experiment (NuBE) will measure the flux of ultra high energy neutrinos $(>10 \mathrm{TeV}$ ) over a $\sim 1 \mathrm{~km}^{2}$ effective area, in coincidence with satellite measured GRB's [10]. It is expected to detect $\approx 20$ events per year, according to the fireball model. Hence, it should be possible to study quantum gravity effects on astrophysical neutrinos that might be observed, or such observations could be used to restrict quantum gravity theories.

Motivated by these interesting posibilities, we have calculated the quantum gravity induced modifications to neutrino propagation [11], using the canonical loop approach.

\section{Loop quantum gravity}

For a recent review of this topic see for example
Ref. [12]. The Ashtekar-Barbero formulation of classical general relativity produces the pair of canonical variables [13]

$$
\left\{A^{i}{ }_{a}(\vec{x}), E_{j}^{b}(\vec{y})\right\}=i \hbar \delta_{j}^{i} \delta_{a}^{b} \delta^{3}(\vec{x}-\vec{y}),
$$

where $A^{i}{ }_{a}(\vec{x})$ is an $S U(2)$ connection and $E_{j}^{b}$ is the inverse densitisized triad. In the connection representation the quantum realization is based upon wave functionals $\Psi\left(A^{i}{ }_{a}\right)$, which must be further annhilated by the constraints: Gauss, diffeomorphism and Hamiltonian.

On the other hand, and in complete analogy with Yang-Mills theories, one can decribe the system in terms of fully gauge invariant quantities. The first step is to introduce the parallel transport matrix

$$
U_{\gamma}\left(s_{1}, s_{2}\right)=\mathcal{P} \exp i \int_{s_{1}}^{s_{2}} A^{i}{ }_{a} \sigma_{i} d x^{a},
$$

where the integration is performed along the open curve $\gamma$. Next, the manifestly $S U(2)$ gauge invariant variables are constructed

$$
\begin{aligned}
& T[\alpha]=\operatorname{Tr} U_{\alpha}, \quad T^{a_{1}, \ldots, a_{n}}[\alpha]\left(s_{1}, \ldots, s_{n}\right)= \\
& =\operatorname{Tr}\left[E^{a_{1}}(1) U_{\alpha}\left(s_{1}, s_{2}\right) \ldots E^{a_{n}}(n) U_{\alpha}\left(s_{n}, s_{1}\right)\right], \\
& \Pi[\gamma]\left(s_{1}, s_{2}\right)=\bar{\psi}\left(\gamma\left(s_{1}\right)\right) U_{\gamma}\left(s_{1}, s_{2}\right) \psi\left(\gamma\left(s_{2}\right)\right),
\end{aligned}
$$

where $\alpha$ is a closed loop. Now, the wave functions are functional of loops and open curves

$$
\Psi\left(\alpha_{1}, \ldots, \alpha_{n}, \gamma_{1}, \ldots, \gamma_{m}\right),
$$

obtained by applying the above operators on a suitable defined vacuum. An orthonormal basis for the loop states, which span the kinematic auxiliary Hilbert space can be constructed using spin networks: the spin network states. Physical wave functions have to further satisfy the constraints. Spin network states are eigenstates of the area and volume operators with finite, discrete eigenvalues. In this way, the theory predicts the discreteness of the space [14].

We will further need a loop state which approximates a flat 3 -metric on $\Sigma$, at scales much larger than the Planck length. For pure gravity this state is called the weave [15]. Flat weave states $|W\rangle$, having a characteristic length $\mathcal{L}$, are constructed by considering collections of Planck 
scale loops. They are meant to be semiclassical states such that if one probes for distances $d>>\mathcal{L}$ the continuous flat classical geometry is regained, while for distances $d<<\mathcal{L}$ the quantum loop structure of space is manifest. In other words, one expects a behavior of the type

$$
\left\langle W\left|\hat{q}_{a b}\right| W\right\rangle=\delta_{a b}+O\left(\frac{\ell_{p}}{\mathcal{L}}\right),
$$

when the mean value of the metric operator is considered. A generalization of such an idea to include matter fields is required. For our analysis it will suffice to exploit the main features that a flat weave with fermions must have. Such a state is denoted by $\mid W, \xi>$, has a characteristic length $\mathcal{L}$ and it is referred to simply as the weave.

\section{The regularization}

A significant progress in the loop approach was made by Thiemann, who put forward a consistent regularization procedure to properly define the quantum Hamiltonian constraint of the full theory [16]. It is based on a triangulation of space with tetrahedra whose sides are of the order $\ell_{P}$. The cornerstone of Thiemann's proposal is the incorporation of the volume operator as a convenient regulator, since its action upon spin network states is finite. We use Thiemann's regularization for the Einstein-Dirac theory, which naturally allows the semiclassical treatment here pursued. The effective Hamiltonian is obtained by considering the expectation value of the fermionic sector of the quantum Hamiltonian constraint, with respect to $|W, \xi\rangle$. Inside this expectation value, operators are expanded around relevant vertices of the triangulation and a systematic approximation is given involving the scales $\ell_{P} \ll$ $\lambda_{D} \ll \lambda_{C}$. Here $\lambda_{D}, \lambda_{C}$ are De Broglie and Compton wavelengths, respectively, of a light fermion. Corrections arise at this level.

The fermionic sector of the Hamiltonian constraint for a two-component spin- $\frac{1}{2}$ field coupled to gravity includes

$$
\begin{aligned}
H^{(1)}(N)= & \int d^{3} x N \frac{E_{i}{ }^{a}}{2 \sqrt{\operatorname{det}(g)}}\left(i \pi^{T} \tau_{i} \mathcal{D}_{a} \xi\right. \\
& + \text { c.c. })
\end{aligned}
$$

plus a Majorana fermion mass term. Besides, it has two further terms: one kinetic-like and the other containing the extrinsic curvature of $\Sigma$ coupled to fermions [16]. Here we discuss the term (2.1) only. Upon regularization, the expectation value $\left\langle W, \xi\left|\hat{H}^{(1)}(N)\right| W, \xi\right\rangle$, which we define as the effective Hamiltoninan $H_{\text {eff }}^{(1)}(N)$, becomes [16]

$$
\begin{aligned}
& H_{\mathrm{eff}}^{(1)}(N)=-\frac{\hbar}{4 \ell_{P}^{4}} \sum_{v \in V(\gamma)} \frac{8 N(v)}{E(v)} \epsilon^{i j k} \epsilon^{I J K}\{\langle W, \xi| \\
& \times \hat{\xi}_{B}\left(v+s_{K}(\Delta)\right) \frac{\partial}{\partial \xi^{A}(v)}\left(\tau_{k} h_{s_{K}(\Delta)}\right)^{A B} \times \\
& \hat{w}_{i I \Delta}(v) \hat{w}_{j J \Delta}(v)|W, \xi\rangle-\langle W, \xi| \tau_{k} \hat{\xi}(v) \frac{\partial}{\partial \xi(v)} \times \\
& \left.\times \hat{w}_{i I \Delta}(v) \hat{w}_{j J \Delta}(v)|W, \xi\rangle-c . c .\right\},
\end{aligned}
$$

where

$$
\hat{w}_{k I \Delta}=\operatorname{Tr}\left(\tau_{k} h_{s_{I}(\Delta)}\left[h_{s_{I}(\Delta)}^{-1}, \sqrt{V_{v}}\right]\right) .
$$

Here $V_{v}$ is the volumen operator, restricted to act upon each vertex $v$. An adapted triangulation to the graph $\gamma$ corresponding to the weave was considered. $\sum_{v \in V(\gamma)}$ runs over all the vertex of the graph $\gamma$.

\section{The calculation}

Next, under the weave expectation value, we expand, around any vertex, all the quantities involved: fermion fields, holonomies, and the operators $\hat{w}_{i I \Delta}(v)$, in powers of the corresponding segments $s^{a}$, having lengths of the order of $\ell_{P}$. The general form of the expectation values is

$$
\begin{aligned}
\sum_{v \in V(\gamma)} & \frac{8 N(v)}{E(v)}<W, \xi \mid \hat{\pi}_{A}(v) \partial_{a} \partial_{b} \ldots \hat{\xi}_{B}(v) \times \\
& \times \hat{R}^{a b \ldots A B}\left(v, s_{I}(v)\right) \mid W, \xi>
\end{aligned}
$$

To proceed with the approximation we think of space as made up of boxes of volume $\mathcal{L}^{3}=d^{3} x$. Each box contains a large number of vertices of the weave $\left(\mathcal{L}>>\ell_{P}\right)$, but is considered infinitesimal in the scale where the space can be regarded as continuous. In successive steps, we take (3.1) to

$$
\sum_{v \in V(\gamma)} \frac{8 N(v)}{E(v)} \pi_{A}(v) \partial_{a} \partial_{b} \ldots \xi_{B}(v)<W, \xi \mid \times
$$




$$
\begin{aligned}
& \hat{R}^{a b \ldots A B}\left(v, s_{I}(v)\right) \mid W, \xi>= \\
& =\sum_{\operatorname{Box}} \pi_{A}(\vec{x}) \partial_{a} \partial_{b} \ldots \xi_{B}(\vec{x}) \sum_{v \in \operatorname{Box}} \ell_{P}^{3} \frac{8 N(v)}{E(v)} \\
& \times<W, \xi\left|\frac{1}{\ell_{P}^{3}} \hat{R}^{A B a b \ldots}\left(v, s_{I}(v)\right)\right| W, \xi>, \\
& =\sum_{\operatorname{Box}} \pi_{A}(\vec{x}) \partial_{a} \partial_{b} \ldots \xi_{B}(\vec{x}) d^{3} x N(\vec{x}) \bar{R}^{A B a b \ldots} \\
& =\int d^{3} x N(\vec{x}) \pi_{A}(\vec{x}) \partial_{a} \partial_{b} \ldots \xi_{B}(\vec{x}) \bar{R}^{A B a b \ldots},
\end{aligned}
$$

where the box-averaged tensor $\bar{R}^{A B a b \cdots}(\vec{x})$ lives in the Lie algebra of $S U(2)$ and it is constructed from $I_{2 \times 2}, \tau_{k}, \delta_{i j}, \epsilon_{i j k}$ and ${ }_{e}^{0} k c$. In order to regain the flat spacetime kinetic term of the fermion Hamiltonian we also demand $|W, \xi\rangle$ to produce the adequate normalization leading to the derivative term in the flat space Dirac equation. By expanding (2.2) at different orders in powers of $s$ one can systematically determine all possible contributions. The order of magnitude of the corresponding expectation values of the gravitational operators is estimated according to

$$
\begin{array}{r}
\left\langle W, \xi\left|\ldots, A_{i a}, \ldots, \sqrt{V_{v}}, \ldots\right| W, \xi\right\rangle \approx \\
\approx \ldots \frac{1}{\mathcal{L}}, \ldots, \ell_{P}^{3 / 2} \ldots
\end{array}
$$

\section{The modified neutrino equation}

¿From the effective Hamiltonian obtained according to the steps described in the previous section, and including the remaining terms in the Hamiltonian cosntraint [17], we obtain the following equation, up to order $\ell_{P}^{2}$

$$
\begin{aligned}
& {\left[i \hbar \frac{\partial}{\partial t}-i \hbar \hat{A} \vec{\sigma} \cdot \nabla+\frac{\hat{C}}{2 \mathcal{L}}\right] \xi(t, \vec{x})+} \\
& \quad+m(\alpha-\beta i \hbar \vec{\sigma} \cdot \nabla) i \sigma_{2} \xi^{*}(t, \vec{x})=0,
\end{aligned}
$$

with

$$
\begin{gathered}
\hat{A}=\left(1+\kappa_{1} \frac{\ell_{P}}{\mathcal{L}}+\kappa_{2}\left(\frac{\ell_{P}}{\mathcal{L}}\right)^{2}+\frac{\kappa_{3}}{2} \ell_{P}^{2} \nabla^{2}\right), \\
\hat{C}=\hbar\left(\kappa_{5} \frac{\ell_{P}}{\mathcal{L}}+\kappa_{6}\left(\frac{\ell_{P}}{\mathcal{L}}\right)^{2}+\frac{\kappa_{7}}{2} \ell_{P}^{2} \nabla^{2}\right), \\
\alpha=1+\kappa_{8} \frac{\ell_{P}}{\mathcal{L}}, \quad \beta=\frac{\kappa_{9}}{2 \hbar} \ell_{P}, \\
\hat{B}=\hat{A}\left(\frac{\hat{C}}{\mathcal{L}}+2 \alpha \beta m^{2}\right) .
\end{gathered}
$$

The dispersion relation corresponding to (4.1) is

$$
\begin{aligned}
E_{ \pm}^{2}(p, \mathcal{L})=\left(A^{2}+m^{2} \beta^{2}\right) p^{2}+ & m^{2} \alpha^{2}+\left(\frac{C}{2 \mathcal{L}}\right)^{2} \\
& \pm B p
\end{aligned}
$$

where $A, B, C$ have been expressed in momentum space. The \pm in Eq. (4.2) stands for the two neutrino helicities. Since typically for neutrinos $\lambda_{C} \gg \lambda_{D}$, our approximation is meaningful only if $\mathcal{L} \leq \lambda_{D}$. In this way we make sure that Eq.(4.1) is defined in a continuous flat spacetime, where the fermionic fields are slowlyvariying. For the purpose of estimating orders of magnitude we take

$$
\mathcal{L}=\lambda_{D}=\frac{1}{\bar{p}},
$$

where $\bar{p}$ is the magnitude of the mean momentum of the neutrino. Up to leading order in $\ell_{P}^{2}$ we obtain

$$
\begin{aligned}
E_{ \pm}(\bar{p})= & \left.E_{ \pm}(p, \mathcal{L})\right|_{p=\bar{p}, \mathcal{L}=1 / \bar{p}} \approx \bar{p}+\frac{m^{2}}{2 \bar{p}}+ \\
& +\ell_{P}\left(\left(\theta_{2} \pm \theta_{4}\right) \bar{p}^{2}+\left(\theta_{1} \pm \theta_{3}\right) m^{2}\right)+ \\
& +\left(\theta_{5} \pm \theta_{6}\right) \ell_{P}^{2} \bar{p}^{3},
\end{aligned}
$$

where we are assuming that all $\theta_{i}$ are non-zero numerical quantities of order one. The velocities are

$$
\begin{aligned}
v_{ \pm}(\bar{p})= & \left.\frac{\partial E_{ \pm}(p, \mathcal{L})}{\partial p}\right|_{p=\bar{p}, \mathcal{L}=1 / \bar{p}}=1-\frac{m^{2}}{2 \bar{p}^{2}}+ \\
& +\kappa_{1}\left(\ell_{P} \bar{p}\right) \mp \kappa_{7} \frac{\left(\ell_{P} \bar{p}\right)^{2}}{2} .
\end{aligned}
$$

\section{Numerical estimations}

An order of magnitude estimation for the modifications to propagation properties arising from the present analysis is obtained by taking the following values for the corresponding neutrino quantities,

$$
\begin{array}{r}
m=10^{-9} \mathrm{GeV}, \quad \bar{p} \sim 10^{5} \mathrm{GeV}, \\
L=10^{10} l y=0.5 \times 10^{42} \frac{1}{\mathrm{GeV}} .
\end{array}
$$

The first interesting parameter is the time delay of neutrinos traveling at velocities $v_{ \pm}$with respect to those traveling at speed $c$

$$
\begin{aligned}
\Delta t_{\nu} & =\frac{L}{c}\left(1-\frac{v_{ \pm}}{c}\right)=\kappa_{1} \frac{L}{c}\left(\bar{p} \ell_{P}\right) \\
& \approx \kappa_{1} 0.3 \times 10^{4} \mathrm{~s} .
\end{aligned}
$$


This number dominates over the delay due to the mass term $\frac{m^{2}}{2 p^{2}}$, which is $\approx 10^{-10} \mathrm{~s}$.

First order corrections to the neutrino dispersion relations which are helicity-independent and linear in the energy, have been also derived in Ref.[18], using a string theory approach. The above expression for the time delay, though helicity independent, has the same form $\sim \ell_{P} \bar{p}$ as the one obtained in Ref.[6].

Finally, the time delay of arrival between the two polarizations is

$$
\begin{aligned}
\Delta t_{ \pm}= & \frac{L}{c^{2}}\left(v_{+}-v_{-}\right)=\kappa_{7} \frac{L}{c}\left(p \ell_{P}\right)^{2} \\
& \approx \kappa_{7} \times 10^{-11} \mathrm{~s} .
\end{aligned}
$$

\section{Acknowledgments}

Partial support is acknowledged from the projects: Conicyt-Conacyt E120-2639 (1997-2-038), Dgapa IN100397, Conacyt 3141PE, Fondecyt 1980806 and Fondecyt 7980018.

\section{References}

[1] G. Amelino-Camelia, J. Ellis, N.E. Mavromatos, D.V. Nanopoulos and S. Sarkar, Nature 393 (1998) 763.

[2] J. Ellis, N.E. Mavromatos and D.V. Nanopoulos, Gen. Rel. Grav. 31 (1999) 1257.

[3] D.V. Ahluwalia, Nature 398 (1999) 199.

G.Z. Adunas, E. Rodriguez-Milla and D.V. Ahluwalia, Probing quantum aspects of gravity, [gr-qc/0006021]; Probing quantum violations of the equivalence principle [gr-qc/0006022]

[4] J. van Paradis et. al., Nature 386 (1997) 686. P.J. Groot et al., IAU Circ. No 6676, 1997. M. L. Metzger et al.,Nature 387 (1997) 878. For a review see for example G.J. Fishman and C.A. Meegan, Ann. Rev. Astron. Astrophys. 33 (1995) 415.

[5] P.N. Bhat, G.J. Fishman, C.A. Meegan, R.B. Wilson, M.N. Brock and W.S. Paclesas, Nature 359 (1992) 217.

[6] R. Gambini and J. Pullin, Phys. Rev. D 59 (1999) 124021.

[7] J. Ellis, N.E. Mavromatos and D.V. Nanopoulos, Probing models of quantum space-time foam, [gr-qc/9909085] and references therein; Gen. Rel. Grav. 32 (2000) 127.
J. Ellis, K. Farakos, N.E. Mavromatos, V.A. Mitsou and D.V. Nanopoulos, Astrophysical J. 535 (2000) 139.

[8] E. Waxman and J. Bahcall, Phys. Rev. Lett. 78 (1997) 2292.

E. Waxman, Nucl. Phys. 87 (Proc. Suppl.) (2000) 345 and references therein.

[9] M. Vietri, Phys. Rev. Lett. 80 (1998) 3690.

[10] M. Roy, H.J. Crawford and A. Trattner, The prediction and detection of UHE Neutrino Bursts, [astro-ph/9903231].

[11] J. Alfaro, H.A. Morales-Técotl and L.F. Urrutia, Phys. Rev. Lett. 84 (2000) 2318.

[12] C. Rovelli, Livings Reviews Vol 1, http://www.livingreviews.org/Articles/

[13] A. Ashtekar, Phys. Rev. Lett. 57 (1987) 2244.

F. Barbero, Phys. Rev. D 51 (1996) 5498.

T. Schilling, [gr-qc/9409031.]

[14] C. Rovelli and L. Smolin, Nucl. Phys. B 442 (1995) 593, ibid. 456 (1995) 753.

A. Ashtekar and J. Lewandowsky Class. and Quant. Grav. 14 (1997) A55; Adv. Theor. Math. Phys. 1 (1998) 388.

[15] A. Ashtekar, C. Rovelli and L. Smolin, Phys. Rev. Lett. 69 (1992) 237.

J. Zeegwaard, Phys. Lett. B 300 (1993) 217.

R. Borissov, Phys. Rev. D 49 (1994) 923.

J. Iwasaki and C. Rovelli, Int. J. Mod. Phys. D 1 (1993) 53 ; Class. and Quant. Grav. 11 (1994) 1653.

J. Iwasaki, Basis states for gravitons in nonperturbative loop representation space [gr-qc/9807013].

[16] T. Thiemann, Class. and Quant. Grav. 15 (1998) 1281;ibid. 15 (1998) 8393; ibid. 15 (1998) 1281.

[17] J. Alfaro, H.A. Morales-Técotl and L.F. Urrutia, in preparation.

[18] J. Ellis, N.E. Mavromatos, D.V. Nanopoulos and V. Volkov, Gravitational recoil effects on fermion propagation in space-time foam, [gr-qc/9911055] 